\title{
Energy expenditure, sex, and endogenous fuel availability in humans
}

\author{
Søren Nielsen, ${ }^{1}$ ZengKui Guo, ${ }^{1}$ Jeanine B. Albu, ${ }^{2}$ Samuel Klein, ${ }^{3}$ Peter C. O’Brien, ${ }^{4}$ \\ and Michael D. Jensen ${ }^{1}$ \\ ${ }^{1}$ Endocrine Research Unit, Mayo Clinic \& Foundation, Rochester, Minnesota, USA \\ ${ }^{2}$ Columbia University, New York, New York, USA \\ ${ }^{3}$ Washington University School of Medicine, St. Louis, Missouri, USA \\ ${ }^{4}$ Department of Biostatistics, Mayo Clinic \& Foundation, Rochester, Minnesota, USA
}

\begin{abstract}
Adipose tissue lipolysis supplies circulating FFAs, which largely meet lipid fuel needs; however, excess FFAs, can contribute to the adverse health consequences of obesity. Because "normal" FFA release has not been well defined, average (mean of 4 days) basal FFA release and its potential regulation factors were measured in 50 lean and obese adults ( 25 women). Resting energy expenditure (REE), but not body composition, predicted most of the interindividual variation in FFA release. There was a significant, positive linear relationship between palmitate release and REE; however, women released approximately $40 \%$ more FFA than men relative to REE. Neither plasma palmitate concentrations nor respiratory quotient by indirect calorimetry differed between men and women. Glucose release rates were not different in men and women whether related to REE or fat free mass. These findings indicate that nonoxidative FFA clearance is greater in women than in men. This could be an advantage at times of increased fuel needs. We conclude that "normal" adipose tissue lipolysis is different in men and women and that the fuel export role of adipose tissue in obesity will need to be reassessed.
\end{abstract}

J. Clin. Invest. 111:981-988 (2003). doi:10.1172/JCI200316253.

\section{Introduction}

Approximately $50 \%$ of postabsorptive energy needs in humans are supplied from the oxidation of fatty acids. The major source of this lipid fuel is circulating FFAs that are released from lipolysis of adipose tissue triglyceride. Maintaining normal FFA availability is of considerable importance in human health (1), because high FFA concentrations are associated with a number of cardiovascular risk factors (2) and a predisposition to type 2 diabetes mellitus (3). Experimentally increasing FFA concentrations in normal humans can induce insulin resistance (4), disordered lipoprotein metabolism (5), altered vascular reactivity (6), and abnormal insulin secretion (7).

At rest, an average adult with $15 \mathrm{~kg}$ of body fat $(>50$ moles of fatty acids) releases less than $0.4 \mathrm{mmol} / \mathrm{min}$ into the circulation to provide energy for fat-free tissue. This is in striking contrast to glucose fuel, where hepatic glycogen stores are approximately $370 \mathrm{mmol}$ and glucose is released at rates of approximately 0.8

Received for publication June 21, 2002, and accepted in revised form January 7, 2003.

Address correspondence to: Michael D. Jensen, Endocrine Research Unit, Joseph 5-194, Mayo Clinic, 200 First Street SW, Rochester, Minnesota 55905, USA. Phone: (507) 255-6768; Fax: (507) 255-4828; E-mail: jensen.michael@mayo.edu.

Conflict of interest: The authors have declared that no conflict of interest exists.

Nonstandard abbreviations used: fat-free mass (FFM); resting energy expenditure (REE); computed tomography (CT); waist-tohip circumference ratio (WHR); General Clinical Research Center (GCRC); coefficient of variation (CV); respiratory quotient (RQ). $\mathrm{mmol} / \mathrm{min}(8)$. Understanding the regulation of this relatively massive adipose tissue fuel depot is important given the prevalence of obesity is increasing rapidly in many Western societies.

The factors that regulate changes in FFA release in response to changing lipid fuel needs are relatively well described. The insulin secretion stimulated by carbohydrate ingestion markedly inhibits FFA release, thereby suppressing FFA concentrations and fatty acid oxidation by $80-90 \%$ (9). In contrast, carbohydrate deprivation or fasting reduces insulin secretion, which enhances adipose tissue FFA release, allowing FFA concentrations to increase by approximately $300 \%$ to facilitate greater fat oxidation. Exercise, the most potent physiological stimulus to fat oxidation, can increase FFA release by more than $300 \%$ (10), primarily through increased catecholamine stimulation of adipose $\beta$-adrenergic receptors. This relatively good understanding of the short-term regulators of FFA release is not mirrored by an equal appreciation of the factors that influence average resting FFA availability.

Understanding the normal interaction between adipose tissue fuel release rates and fat-free mass (FFM) fuel use rates is important for understanding the effects of body fat on health. The approach to comparing fuel release rates between groups with different weights and/or different body composition can influence how the information is interpreted, however. For example, there has been controversy regarding whether obesity causes higher FFA concentrations because of greater release or lesser clearance of FFA from the circulation. Obese adults have FFA release rates similar to lean adults, relative to body weight or body fat $(11,12)$, 
but when compared on an absolute basis (micromoles per minute) or relative to $\operatorname{FFM}(12,13)$, FFA release is greater in obesity. Therefore, understanding whether higher concentrations are due to increased release or reduced uptake becomes a matter of perspective. The current approach to this issue, derived from early studies of FFA kinetics (14-17) is that FFM is the optimal denominator, although the use of resting energy expenditure (REE) was at one time examined (11). Unfortunately, this long-held convention originates from data derived from relatively small numbers of subjects $(12,14,15,17)$, sometimes of a single sex (16), and on an admittedly empirical basis (15).

An inherent problem in comparing lean and obese men and women using the approach of dividing FFA release rates (micromoles per minute) by FFM is that it assumes a mathematical relationship between FFA release and FFM known as "ratio standard" (a slope of 1.0 and an intercept of 0 ). This assumption may lead to serious interpretive errors if it is not correct (18). Whether the association between FFA release and body size or metabolic rate is altered after accounting for sex, body fat characteristics, and hormone concentrations have not been examined previously. Another supposition is that men and women, because they have similar FFA concentrations, can be compared using the same approaches. If these two precepts are not correct, the manner in which fuel storage and release are examined will need to be reconsidered. Because the primary function of FFA is to serve as an oxidative substrate, we hypothesized that REE, rather than FFM, would be a better index for judging whether adipose tissue FFA release rates are appropriate. Moreover, variability in the relationship between FFM and REE (19) and the effects of sex on FFM and REE (20) underscores the importance of evaluating this fundamental, biological issue. These experiments tested the hypothesis that resting, postabsorptive FFA release rates are more strongly related to REE than to body composition and examined whether sex, body fat characteristics, or hormone concentrations modify this relationship.

\section{Methods}

Subjects. Fifty research volunteers (25 men and 25 women) participated in this Institutional Review Board-approved study after written, informed consent was obtained. All participants were nonsmokers, not taking medications, and had been weight stable for the previous 3 months. All had a normal oral glucose tolerance test prior to entering the study and had normal blood pressure, hematological indices, liver and renal function. All women were premenopausal. Subjects were recruited such that approximately one-half of the men and women were lean and one-half were obese and that a wide range of body fat distribution was included within the obese group.

Experimental design. Because body composition is a potentially important determinant of fuel metabolism, it was assessed in several ways. These included the fol- lowing: (a) dual-energy x-ray absorptiometry (Lunar Radiation Corp., Madison, Wisconsin, USA) to measure total body fat and FFM (21); (b) a single-slice computed tomography (CT) scan (GE-Imatron Inc., San Francisco, California, USA) of the abdomen at the $\mathrm{L}_{2-3}$ level (22) to assess central fat accumulation (intraabdominal and abdominal subcutaneous fat); (c) the waist-to-hip circumference ratio (WHR) to evaluate relative body fat distribution; (d) needle-liposuction adipose tissue biopsies were performed to determine abdominal subcutaneous and gluteal fat cell size. Procedures for the measurement of fat cell size were followed according to the methods of Di Girolamo et al. (23). At least 200 cells per site were sized to provide an estimate of average fat cell diameter. Average fat cell diameter was then used to calculate fat cell volume and lipid content (23). BMI was calculated as body weight (kilograms) per height (meters squared).

All participants were provided weight-maintaining meals ( $40 \%$ carbohydrates, $40 \%$ fat, $20 \%$ protein) in the Mayo Clinic General Clinical Research Center (GCRC) for 2 weeks prior to the studies, including the 4 study days. At the end of the 2-week period participants were admitted to the GCRC for four consecutive overnight stays. During the study days the volunteers were allowed to perform their usual activities (including work), but received their meals in the GCRC, including the last meal, which was given at 1800 hours, and a snack that was provided at 2100 hours. REE was measured by indirect calorimetry (DeltaTrac Metabolic Cart; Sensormedics Inc., Yorba Linda, California, USA) each morning before the participants arose from bed. The metabolic cart was calibrated each morning prior to the study, and additional quality control for the carts included monthly pressure calibrations and gas calibrations together with biannual calibrations of the metabolic carts using an alcohol burn test.

Samples for the measurements of postabsorptive steady-state palmitate and glucose release rates and plasma hormone and catecholamine concentrations (insulin, growth hormone, epinephrine, and norepinephrine) were obtained between 0700 and 0730 hours each day. The FFA and glucose release rates into the circulation were measured using continuous intravenous infusions of isotopically labeled $\mathrm{FFA}\left(\left[\mathrm{U}-{ }^{13} \mathrm{C}\right]\right.$ palmitate $)$ (24) and glucose $\left(\left[6-{ }^{2} \mathrm{H}_{2}\right]\right.$ glucose $)(25)$, respectively. The needed preservative was not added to the catecholamine collection tubes for three volunteers' studies, and thus no measures of epinephrine or norepinephrine were available for them. The CT image from one volunteer was unable to be retrieved for analysis.

Four studies were performed because of the need to more accurately define average, resting FFA release given the substantial (coefficient of variation of $\sim 30 \%$ ) day-to-day intraindividual variability in resting FFA kinetics (26). By more accurately defining the relationship between average FFA availability and REE (or body composition) for each participant we improved the ability to test for other variables (such as body fat or 
Table 1

Clinical characteristics, body composition, and hormone concentrations of the men and women participating in this study

\begin{tabular}{|c|c|c|}
\hline & Women $(n=25)$ & $\operatorname{Men}(n=25)$ \\
\hline Age (years) & $30(19-46)$ & $26(21-48)$ \\
\hline $\operatorname{BMI}\left(\mathrm{kg} / \mathrm{m}^{2}\right)$ & $24.3(18.9-35.3)$ & $26.9(20.1-36.0)$ \\
\hline WHRA & $0.80(0.70-0.97)$ & $0.87(0.79-1.02)$ \\
\hline Body fat $(\%)^{A}$ & $38(27-52)$ & $24(8-36)$ \\
\hline Total fat mass $(\mathrm{kg})^{\mathrm{A}}$ & $23.5(13.9-52.8)$ & $20.4(5.1-40.8)$ \\
\hline $\mathrm{FFM}(\mathrm{kg})^{\mathrm{A}}$ & $42.1(34.8-55.2)$ & $67.4(52.7-77.5)$ \\
\hline Visceral fat area $\left(\mathrm{cm}^{2}\right)^{\mathrm{A}}$ & $45(21-174)$ & $83(27-274)$ \\
\hline Abdominal subcutaneous fat area $\left(\mathrm{cm}^{2}\right)$ & $162(60-527)$ & $115(40-404)$ \\
\hline Abdominal fat cell ( $\mu$ g lipid/cell) & $0.493(0.178-1.084)$ & $0.584(0.070-1.168)$ \\
\hline Gluteal fat cell ( $\mu$ g lipid/cell) & $0.527(0.304-0.824)$ & $0.454(0.215-0.829)$ \\
\hline Insulin $(\mathrm{pmol} / \mathrm{I})$ & $48 \pm 18$ & $48 \pm 19$ \\
\hline Norepinephrine (nmol/l) & $103 \pm 24$ & $98 \pm 21$ \\
\hline Epinephrine (pmol/l) & $16 \pm 5$ & $19 \pm 9$ \\
\hline Growth hormone $(\mu \mathrm{g} / \mathrm{l})^{\mathrm{A}}$ & $4.97 \pm 3.51$ & $2.65 \pm 1.09$ \\
\hline Palmitate release rate $(\mu \mathrm{mol} / \mathrm{min})$ & $89 \pm 27$ & $87 \pm 24$ \\
\hline Palmitate concentration $(\mu \mathrm{mol} / \mathrm{I})$ & $92 \pm 23$ & $84 \pm 19$ \\
\hline $\operatorname{REE}(\mathrm{kcal} / \text { day })^{\mathrm{A}}$ & $1,558 \pm 222$ & $1,880 \pm 206$ \\
\hline RQ & $0.82 \pm 0.04$ & $0.83 \pm 0.04$ \\
\hline
\end{tabular}

Values are given as mean $\pm S D$ if the data were normally distributed or median (range) if the data were not normally distributed. The plasma hormone and catecholamine concentrations are the mean of all samples (four to five samples taken each day for 4 study days). Likewise, the palmitate release rates, REE, and RQ data from each volunteer are the mean values of 4 study days. ${ }^{A} P<0.01$ men vs. women.

plasma hormone concentrations) that might also influence FFA release rates.

Palmitate was chosen as a representative fatty acid to allow assessment of FFA kinetics because it is one of the major FFAs (29\% $\pm 3 \%$ in these volunteers) and because its metabolic properties are representative of the remaining seven major FFAs (26). Palmitate was the same proportion of FFA in men and women. Because circulating glucose kinetics are less variable from day to day (27), they were measured on 2 of the 4 days.

Analysis of samples. Plasma palmitate enrichment was measured using gas chromatography/combustion/isotope-ratio mass spectrometry as described (24). Plasma glucose enrichment was measured using gas chromatography/mass spectrometry (25). Insulin and growth hormone concentrations were measured using chemiluminescent sandwich assays (Sanofi Diagnostics Pasteur Inc., Chaska, Minnesota, USA), and catecholamines were measured using HPLC with electrochemical detection (28).

Calculations. Steady-state appearance (and disappearance) rates of palmitate and glucose were calculated using the mean enrichment values and tracer infusion rates as described previously $(24,25)$.

Statistical analysis. The average palmitate release rate (micromoles per minute) was used as the dependent variable in a multivariate, stepwise regression analysis using the following candidate independent variables: average REE, sex, FFM, fat mass, percentage of fat, intraabdominal fat area, abdominal fat cell size, and average insulin, growth hormone, and catecholamine concentrations. Variables that were not normally distributed (such as plasma epinephrine concentrations) were log transformed in order to be appropriately tested for inclusion in the model. Using the approach of stepwise variable selection, stepping up, only variables with a significance level of 0.05 were included in the model. (Using a looser criterion of $P=0.10$, the model was the same.) We also tested quadratic terms and two-way interactions among variables in the final model and found none significant. To ensure that FFM and total fat mass were not prematurely excluded from consideration, they were included in a non-stepwise model that also included the variables found to be significant in the stepwise model. Exploratory univariate regression analyses were performed for the purposes of comparing the relative strengths of associations between palmitate release rates and alternative normalizing variables and for the purposes of displaying factors that could explain the residual variance in palmitate rates. Glucose release rates (milligrams per minute) were examined using linear regression analysis versus REE and FFM, with an additional multivariate analysis to test for a sex effect. Likewise, multivariate regression analysis was used to determine whether there was a sex effect on the relationship between FFM and REE.

\section{Results}

Subject characteristics. The characteristics of the subjects are provided in Table 1. The expected differences between men and women in body composition, body fat distribution, and REE were observed. Plasma palmitate concentrations were not different, and with the exception of the expected greater concentrations of growth hormone in women than in men $(P<0.005)$, plasma hormone and catecholamine concentrations were not different. The relationship between REE and

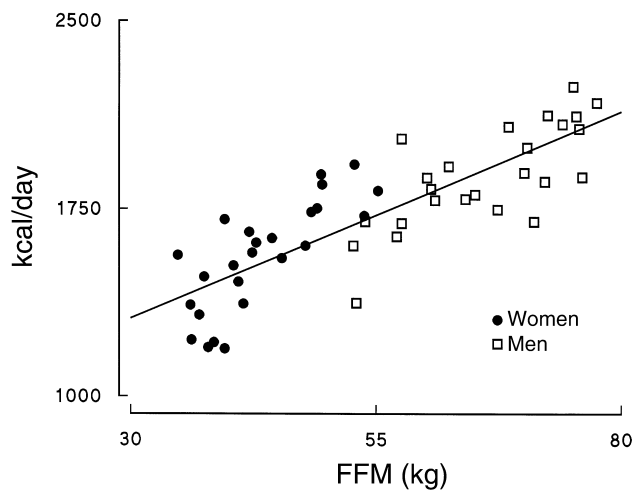

Figure 1

REE is plotted vs. FFM for the men and women participating in the study. 


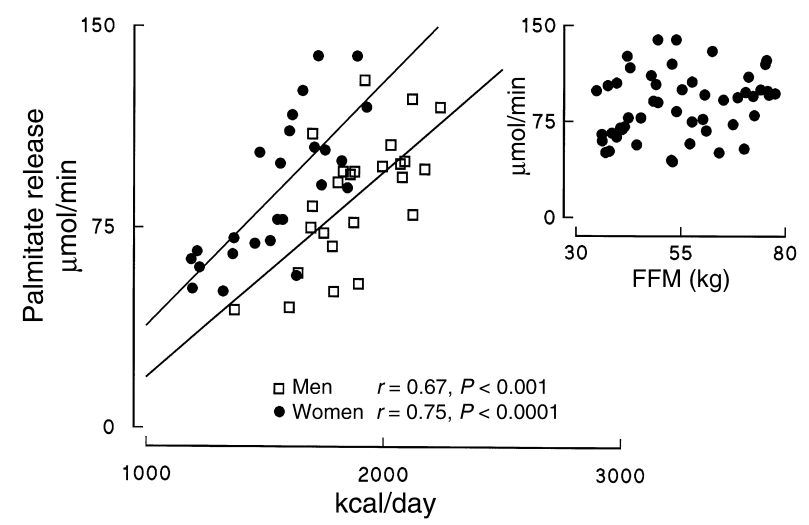

Figure 2

Resting postabsorptive palmitate release rate is plotted vs. REE for the men and women participating in the study. The inset depicts the relationship between palmitate release and FFM for these same volunteers.

FFM was assessed using multiple linear regression analysis using a sex and a sex $\times$ FFM interaction term. This relationship was not different between men and women (Figure 1; simple $r^{2}=0.69, P<0.0001$ ).

Day-to-day variability of indirect calorimetry and substrate measurements. The coefficient of variation $(\mathrm{CV})$ for REE was $4 \% \pm 2 \%$ and for the respiratory quotient (RQ) was $3 \% \pm 1 \%$. Perhaps thanks to the greater dietary control imposed by the study design, the CVs for average fasting plasma palmitate concentrations and flux were $16 \% \pm 8 \%$ and $14 \% \pm 8 \%$ (which is approximately onehalf of what we have observed previously) (26). The CV for plasma glucose concentrations over the 4 days was $3 \% \pm 1 \%$, whereas the difference between glucose flux between the 2 study days for which it was measured averaged $13 \% \pm 13 \%$.

Relationship between FFA release and REE versus FFM. For the entire group, palmitate release rates were positively correlated with REE $(r=0.54, P<0.0001)$, but were not significantly correlated with FFM (see inset of Figure 2; $r=0.25, P=0.09$ ). Because the multivariate regression analysis (see below) indicated a significant sex effect, palmitate release was plotted in relationship to REE separately for men and women (Figure 2) and sex-specific univariate regression analyses were performed. The relationship between palmitate kinetics and REE was stronger for each sex than for the combined group. Palmitate release rates relative to REE were significantly greater in women than in men, such that for a given level of energy expenditure, the average fuel availability from FFA was approximately $40 \%$ more in women. Note that the greater FFA release rates were not associated with higher plasma FFA (palmitate) concentrations in women (Table 1). Nor were these sex differences in FFA release associated with differences in resting fuel use; the RQ, which provides a measure of the proportion of fat and carbohydrate being oxidized, was the same in men and women (Table 1).

The initial multivariate stepwise regression analysis results disclosed that REE, sex, and plasma epinephrine concentrations were significant predictors of average palmitate flux. The parameter estimates for the model are provided in Table 2. The $r^{2}$ for this model was 0.57. Percentage of body fat and indices of central fat distribution (visceral fat area and WHR) were not included in the final model.

To ensure variables were not inappropriately left out of the model by the stepwise regression analysis approach we performed regression analysis using a nonstepping model that included REE, sex, FFM, fat mass, and our measures of adrenergic tone (plasma epinephrine and norepinephrine concentrations) as independent variables. The results were the same as for the stepwise model. The $r^{2}$ for this model was 0.61 . The parameter estimates and associated $P$ values for this model are provided in Table 3 . Consistent with the stepwise approach, REE, sex, and epinephrine significantly contributed to the ability to predict the interindividual differences in palmitate flux. Total fat mass, FFM, and plasma norepinephrine did not contribute significantly to this model. Although the parameter estimate for REE appears small, the REE values are expressed in the table per $100 \mathrm{kcal} /$ day (range of values $1,190-2,238 \mathrm{kcal} /$ day), creating a numerically small parameter estimate that was highly statistically significant. The parameter estimate for male sex was -31 , consistent with the visual separation of the regression lines for men and women depicted in Figure 2. The parameter estimate for plasma epinephrine should be interpreted in the context of the need to log transform the plasma epinephrine concentrations because of their skewed distribution.

Despite the good relationship between REE and palmitate release, there remained individual variation from the sex-specific group relationships (Figure 2). Because the multivariate regression analysis indicated that epinephrine contributed to the interindividual differences in palmitate release rates relative to REE, we examined this interaction in a different manner to allow a better visualization of the interactions. To do this the residual variance in palmitate release was determined for each participant. This value represents how each participant's average palmitate release rate differed from their sex-specific group and is calculated by

\section{Table 2}

Parameter estimates for stepwise (stepping up) multivariate regression analysis

\begin{tabular}{lccc}
\hline Variable & Parameter estimate & Standard error & $P$ value \\
Intercept & -91 & 28 & 0.0019 \\
REE & 8.8 & 1.2 & $<0.0001$ \\
Male & -32 & 6 & $<0.0001$ \\
Plasma epinephrine $(\log )$ & 16 & 7 & 0.0259
\end{tabular}

The statistically significant parameter estimates for the relationship between palmitate flux $(\mu \mathrm{mol} / \mathrm{min}$ - dependent variable $)$ and the independent variables listed in Methods are provided. REE is given as per $100 \mathrm{kcal} / \mathrm{day}$; plasma epinephrine concentrations were log transformed for inclusion in the model because of the skewed distribution of the values. 
Table 3

Parameter estimates for nonstepped multivariate regression analysis

$\begin{array}{lccc}\text { Variable } & \text { Parameter estimate } & \text { Standard error } & P \text { value } \\ \text { Intercept } & -105 & 32 & 0.0023 \\ \text { REE } & 6.9 & 2.1 & 0.0024 \\ \text { Male } & -31 & 14 & 0.0356 \\ \text { FFM }(\mathrm{kg}) & 0.391 & 0.650 & 0.5507 \\ \text { Fat mass }(\mathrm{kg}) & 0.366 & 0.316 & 0.2539 \\ \text { Plasma epinephrine }(\log ) & 16 & 7 & 0.0275 \\ \text { Plasma norepinephrine } & 0.15 & 0.12 & 0.2290\end{array}$

The parameter estimates for the formula describing the relationship between palmitate flux ( $\mu \mathrm{mol} / \mathrm{min}$ - dependent variable) and the following independent variables: REE (given as per $100 \mathrm{kcal} /$ day); male sex; FFM; fat mass; log plasma epinephrine concentration; plasma norepinephrine concentration.

subtracting the predicted palmitate release (based on REE and sex-specific regression formulas) from the observed palmitate release for each subject.

Consistent with the multivariate regression analysis, the mean fasting plasma epinephrine concentrations were positively correlated $(r=0.33, P<0.05)$ with residual palmitate release. Although plasma norepinephrine concentrations were not a significant contributor to the model, they were also positively correlated $(r=0.31, P<0.05)$ with residual palmitate release. There was no significant correlation between plasma epinephrine and norepinephrine concentrations. Neither the mean fasting plasma insulin or growth hormone concentrations were correlated with the residual variance in palmitate release.

Although indices of body fatness were not significant predictors of palmitate flux in the regression models, we have found previously that upper-body obesity is associated with elevated FFA concentrations and flux (13). We therefore explored whether visceral fat area might be associated with the residual variance in palmitate flux. In this analysis the residual variance in palmitate release was correlated $(r=0.44, P=0.03)$ with visceral fat area in men (Figure 3). Percentage of body fat and WHR tended ( $P=0.06$ and $P=0.07$, respectively) to be positively correlated with residual variance in palmitate release in men. To our surprise, abdominal fat cell size did not significantly correlate $(r=0.24, P=0.24)$ with residual palmitate flux in men. None of the body fat parameters (percentage of body fat, kilograms of body fat, WHR, visceral or abdominal subcutaneous fat area, or gluteal or abdominal subcutaneous fat cell size) correlated with residual palmitate release rates in women.

If REE is important for determining what constitutes "normal" FFA release rates, we anticipated that plasma palmitate concentrations would vary proportionately with the residual variance in palmitate release relative to REE. Indeed, there was a strong, positive correlation between residual palmitate release and palmitate concentrations (Figure 4). Thus, individuals in whom palmitate release rates were greater than their sex-specific group relationship had higher plasma concentrations and vice versa.
Glucose appearance rates. The sex difference in the relationship between fuel mobilization and REE was specific to FFA. Glucose release rates correlated directly with both FFM $(r=0.71, P<0.00001)$ and REE $(r=0.62, P<0.0001)$. Multivariate regression analysis failed to disclose a sex effect on the relationship between glucose release rates and FFM or REE (Figure $5)$. The relationship between glucose appearance and REE in women $(r=0.50)$ was glucose flux $(\mathrm{mg} / \mathrm{min})=$ $70+[\operatorname{REE}(\mathrm{kcal} /$ day $) \times 0.034]$; in men $(r=0.34)$ was glucose flux $=82+[\operatorname{REE}(\mathrm{kcal} /$ day $) \times 0.039]$. The $95 \%$ confidence intervals for both the intercept and the slope overlapped for men and women.

\section{Discussion}

These studies were conducted to address two previously unsettled issues with respect to the relationship between adipose tissue fuel release and lean tissue fuel use. Our first concern was that the traditional means of comparing resting FFA release rates between groups - dividing by FFM - is inappropriate and misleading. The second major concern was the lack of information as to whether men and women could be compared using the same approach. In addition, by more accurately determining the average basal FFA release rate for each individual, we were able to examine additional factors that might relate to basal FFA release. Consistent with our hypothesis, REE was found to be a better predictor of basal palmitate release than FFM. Our inability to detect an association with FFM after adjusting for other variables could be because any independent relationship between FFA release and FFM was too slight to detect, rather than because there is no relationship at all. Our second concern, that comparing men and women directly could lead to problems in identifying "abnormal" FFA release, was also confirmed. The greater relative FFA release rates in women despite similar plasma concentrations can only be
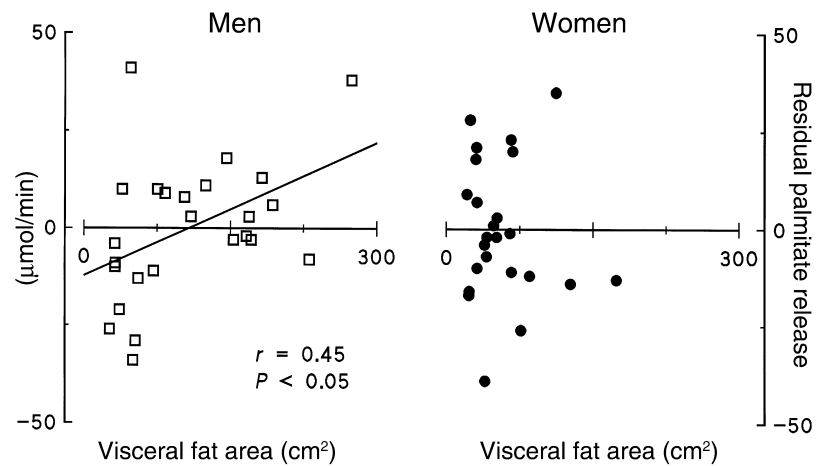

\section{Figure 3}

The relationship between visceral fat area and residual differences between sex-specific REE/palmitate release (flux) rate relationships for men and women are shown. In men visceral fat area showed a significant, positive relationship $(r=0.45, P<0.05)$ with the residual palmitate flux values. In women visceral fat area was not correlated with the residual palmitate flux values; neither were percentage of body fat, WHR, or fat cell size. 


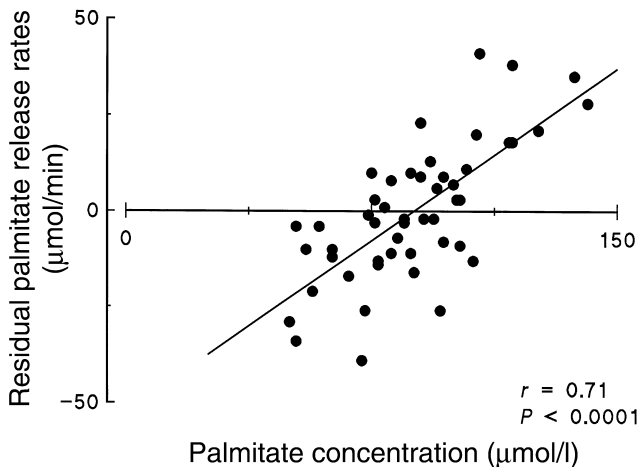

Figure 4

The relationship between plasma palmitate concentration and residual differences between sex-specific REE/palmitate release rate relationships for all volunteers.

explained by greater clearance rates. Thus, understanding adipose tissue lipolysis will require that energy expenditure and sex, as well as body composition, be taken into account.

Despite the sex differences in FFA release and uptake there was no difference between men and women in FFA concentrations or in the proportion of energy expenditure accounted for by fatty acid oxidation. This is possible because not all FFAs removed from the circulation are immediately oxidized - significant proportions are re-esterified as tissue triglyceride (29). It follows that at rest women shunt more circulating FFA into re-esterification pathways than do men. Recent studies (30) have shown that lean women have almost double the VLDL-triglyceride production rates as lean men, despite comparable the VLDL-triglyceride concentrations. Thus, greater hepatic clearance, re-esterification, and export of the "excess" FFA back to adipose tissue could explain our findings.

This basic, physiological difference in FFA metabolism between men and women could be advantageous at times of increased energy requirements, such as exercise or stress. For example, adipose tissue lipolysis increases somewhat gradually in response to exercise, whereas energy expenditure and fat oxidation increase almost immediately $(10,31)$. Women should, therefore, be able to increase the proportion of FFA directed toward oxidation (with a concomitant reduction in FFA re-esterification) and better match circulating lipid fuel availability to lipid fuel needs as energy expenditure increases. The combination of greater availability and greater clearance may explain the ability of women to oxidize relatively more fat than do men during exercise (32). This greater resting lipid fuel release rate is accomplished without exposing tissues to higher and potentially harmful FFA concentrations.

Excess accumulation of central fat is associated with adverse health consequences (33), which have been linked to higher FFA concentrations (1). In this study, indices of body fatness did not contribute significantly to FFA release rates after REE, sex, and epinephrine were considered. The number of individuals with upper-body obesity was small, however, compared with our previous studies (13). As a way to assess whether the results of the present study were in conflict with previous studies we examined whether visceral fat area was associated with greater palmitate release rates after interindividual differences in REE were taken into account. There was an association with visceral adiposity in men, but we did not detect such an effect in women. We reported previously that FFA flux is increased in upper-body obesity when larger numbers of lean and obese women (matched for FFM and REE) were evaluated (13). Therefore, we do not believe that the current data should be taken to indicate that centrally obese adults have the same rates of lipolysis as do lean adults. The narrower range of visceral fat area in women compared with men in the present study (Table 1) may have limited our ability to detect an effect of visceral fat on FFA kinetics.

One cannot infer causal mechanisms from statistical analysis alone: that interindividual variances in REE drive FFA release rates. It might be argued that differences in FFA release rates determine REE. A number of observations lead us to doubt that FFA modulates REE, however. For example, experimental increases in plasma FFA do not raise REE (34). In addition, REE is not reduced by suppression of FFA release and fatty acid oxidation using acipimox (35) or insulin (36), or by pharmacological blockade of fatty acid oxidation (37). We conclude differences in REE seem to be the major, underlying factor influencing the rate at which adipose tissue releases its fuel into the circulation in resting humans. Of note, the intercept of the relationship between REE and FFA release rates was not zero. This indicates that dividing FFA release rates by a measure of energy expenditure is inappropriate. Regression analysis will be needed to compare groups with different mean REE values.

It is not known how REE influences adipose tissue fuel export function in the basal state and why this

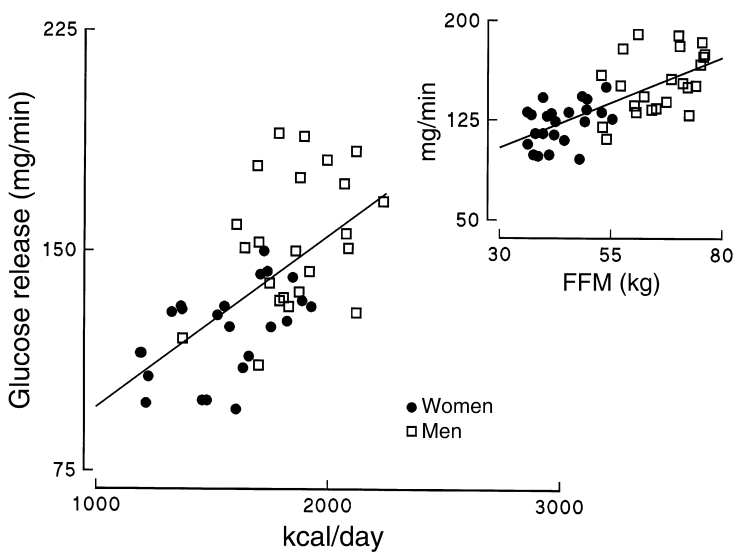

Figure 5

Resting postabsorptive glucose release rate is plotted vs. REE for the men and women participating in the study. The inset depicts the relationship between glucose release and FFM for these same volunteers. 
relationship is different in men and women. However, interpreting the present data within the framework of previously published studies, we would like to suggest a new concept in the regulation of adipose tissue lipolysis. Plasma FFA concentrations may themselves serve as a signal for the secretion of hormones that modulate lipolytic activity. Insulin, catecholamines, and growth hormone exert rapid effects on adipose tissue lipolysis and are the major regulators of systemic FFA release. High-plasma FFA concentrations enhance glucose-stimulated insulin secretion (38), which can then feed back to inhibit lipolysis. High FFA also suppresses growth hormone secretion (39), which in turn can reduce lipolysis. There is also evidence that FFAs may themselves directly inhibit adipose tissue FFA release (40). In contrast, lowering plasma FFA concentrations decreases glucose-mediated insulin secretion (41) and can increase plasma growth hormone concentrations (42). Therefore, changes in plasma FFA concentrations influence the secretion of lipolytic and antilipolytic hormones in a counterregulatory fashion. The data from the present study suggest to us that energy expenditure (requirement) is an indicator of the need for FFA as an oxidative fuel. In general, even when individuals are in energy balance, greater energy requirements require greater plasma FFA uptake and oxidation, thereby lowering plasma FFA concentrations and setting in motion counterregulatory responses that maintain plasma FFA. Likewise, lesser energy requirements and fatty acid oxidation would require lesser FFA uptake and thus reduced lipolysis to maintain "normal" plasma FFA concentrations. This feedback loop provides a mechanism that allows energy demand to regulate the release of FFA, an oxidative fuel, into the circulation. The higher basal rate of lipolysis we observed in women can be explained by a higher rate of nonoxidative FFA clearance in women than in men.

In summary, these results have important implications for understanding endogenous fuel mobilization and how it may affect human health. We found that (a) FFA release rates are highly correlated with REE; (b) women have higher rates of FFA release in relationship to energy requirements than do men; (c) plasma FFA concentrations are not different in women and men, indicating greater nonoxidative FFA clearance in women; and (d) increased basal catecholamine concentrations are associated with greater resting FFA release rates. These findings suggest that new approaches will be required to assess how variations in lipolytic activity might impact on obesityrelated disease. The study of adipose tissue lipolytic rates should account for REE and sex if the goal is to identify abnormalities in the regulation of adipose tissue lipolysis. The greater relative FFA availability in women compared with men at comparable FFA concentrations could be a distinct metabolic advantage at times of increasing needs for lipid fuel, such as during exercise, stress, or starvation.

\section{Acknowledgments}

Supported by grants DK-40484, DK-50456, DK37948, and RR-0585 from the U.S. Public Health Service, and the Danish Medical Research Council and the Mayo Foundation. We acknowledge the assistance of Claudia Powel and Stephen Weigand for statistical analysis and Rita Nelson and Carol Siverling for laboratory technical assistance.

1. Unger, R.H. 1995. Lipotoxicity in the pathogenesis of obesity-dependent NIDDM. Diabetes. 44:863-869.

2. Bjorntorp, P. 1990. "Portal" adipose tissue as a generator of risk factors for cardiovascular disease and diabetes. Arteriosclerosis. 10:493-496.

3. Paolisso, G., et al. 1995. A high concentration of fasting plasma nonesterified fatty acids is a risk factor for the development of NIDDM. Diabetologia. 38:1213-1217.

4. Ferrannini, E., Barrett, E.J., Bevilacqva, S., and DeFronzo, R.A. 1983. Effect of fatty acids on glucose production and utilization in man. J. Clin. Invest. 72:1737-1747.

5. Lewis, G.F., Uffelman, K.D., Szeto, L.W., Weller, B., and Steiner, G. 1995. Interaction between free fatty acids and insulin in the acute control of very low density lipoprotein production in humans. J. Clin. Invest. 95: $158-166$.

6. Steinberg, H.O., et al. 1997. Elevated circulating free fatty acid levels impair endothelium-dependent vasodilation. J. Clin. Invest. 100:1230-1239.

7.Zhou, Y.P., and Grill, V.E. 1994. Long-term exposure of rat pancreatic islets to fatty acids inhibits glucose-induced insulin secretion and biosynthesis through a glucose fatty acid cycle. J. Clin. Invest. 93:870-876.

8. Taylor, R., et al. 1996. Direct assessment of liver glycogen storage by ${ }^{13} \mathrm{C}$ nuclear magnetic resonance spectroscopy and regulation of glucose homeostasis after a mixed meal in normal subjects. J. Clin. Invest. 97:126-132.

9. Jensen, M.D. 1995. Gender differences in regional fatty acid metabolism before and after meal ingestion. J. Clin. Invest. 96:2297-2303.

10. Kanaley, J.A., Mottram, C.D., Scanlon, P.D., and Jensen, M.D. 1995. Fatty acid kinetic responses to running above or below lactate threshold. J. Appl. Physiol. 79:439-447.

11. Issekutz, B., Jr., Bortz, W.M., Miller, H.I., and Paul, P. 1967. Turnover rate of plasma FFA in humans and in dogs. Metabolism. 16:1001-1009.

12. Bjorntorp, P., Bergman, H., and Varnauskas, E. 1969. Plasma free fatty acid turnover rate in obesity. Acta. Med. Scand. 185:351-356.

13. Jensen, M.D., Haymond, M.W., Rizza, R.A., Cryer, P.E., and Miles, J.M. 1989. Influence of body fat distribution on free fatty acid metabolism in obesity. J. Clin. Invest. 83:1168-1173.

14. Bjorntorp, P., Bergman, H., Varnauskas, E., and Lindholm, B. 1969. Lipid mobilization in relation to body composition in man. Metabolism. 18:841-851.

15. Birkenhäger, J.C., and Tjabbes, T. 1969. Turnover rate of plasma FFA and rate of esterification of plasma FFA to plasma triglycerides in obese humans before and after weight reduction. Metabolism. 18:18-32.

16. Hagenfeldt, L. 1975. Turnover of individual free fatty acids in man. Fed. Proc. 34:2246-2249.

17. Nestel, P.J., and Whyte, H.M. 1968. Plasma free fatty acid and triglyceride turnover in obesity. Metabolism. 17:1122-1128.

18. Tanner, J.M. 1949. Fallacy of per-weight and per-surface area standards, and their relation to spurious correlation. J. Appl. Physiol. 2:1-15.

19. Bogardus, C., et al. 1986. Familial dependence of the resting metabolic rate. N. Engl. J. Med. 315:96-100.

20. Ferraro, R., et al. 1992. Lower sedentary metabolic rate in women compared with men. J. Clin. Invest. 90:780-784.

21. Jensen, M.D., et al. 1993. Assessment of body composition with use of dual-energy $x$-ray absorptiometry: evaluation and comparison with other methods. Mayo Clin. Proc. 68:867-873.

22. Jensen, M.D., Kanaley, J.A., Reed, J.E., and Sheedy, P.F. 1995. Measurement of abdominal and visceral fat with computed tomography and dual-energy x-ray absorptiometry. Am. J. Clin. Nutr. 61:274-278.

23. Di Girolamo, M., Mendlinger, S., and Fertig, J.W. 1971. A simple method to determine fat cell size and number in four mammalian species. Am. J. Physiol. 221:850-858.

24. Guo, Z., Nielsen, S., Burguera, B., and Jensen, M.D. 1997. Free fatty acid turnover measured using ultralow doses of $\left[\mathrm{U}-{ }^{13} \mathrm{C}\right]$ palmitate. J. Lipid Res. 38:1888-1895.

25. Kury, D., and Keller, U. 1991. Trimethylsilyl-O-methyloxime derivatives for the measurement of [6,6-2H2]-D-glucose-enriched plasma samples by gas chromatography-mass spectrometry. J. Chrom. 572:302-306.

26. Jensen, M.D., Caruso, M., Heiling, V., and Miles, J.M. 1989. Insulin regulation of lipolysis in nondiabetic and IDDM subjects. Diabetes. 38:1595-1601. 
27. Wolfe, R.R., Allsop, J.R., and Burke, J.F. 1979. Glucose metabolism in man: responses to intravenous glucose infusion. Metabolism. 28:210-220.

28. Causon, R.C., Carruthers, M.E., and Rodnight, R. 1981. Assay of plasma catecholamines by liquid chromatography with electrochemical detection. Anal. Biochem. 116:223-226.

29. Klein, S., and Wolfe, R.R. 1990. Whole-body lipolysis and triglyceridefatty acid cycling in cachectic patients with esophageal cancer. J. Clin. Invest. 86:1403-1408.

30. Mittendorfer, B., Patterson, B.W., and Klein, S. 2003. Effect of sex and obesity on very-low density lipoprotein-triglyceride kinetics. Am. J. Clin. Nutr. 77:573-579.

31. Kanaley, J.A., Cryer, P.E., and Jensen, M.D. 1993. Fatty acid kinetic responses to exercise. Effects of obesity, body fat distribution, and energy-restricted diet. J. Clin. Invest. 92:255-261.

32. Tarnopolsky, M.A., Atkinson, S.A., Phillips, S.M., and MacDougall, J.D. 1995. Carbohydrate loading and metabolism during exercise in men and women. J. Appl. Physiol. 78:1360-1368.

33. Kissebah, A.H., et al. 1982. Relation of body fat distribution to metabolic complications of obesity. J. Clin. Endocrinol. Metab. 54:254-260.

34. Boden, G., et al. 1991. Effects of fat on insulin-stimulated carbohydrate metabolism in normal men. J. Clin. Invest. 88:960-966.

35. Segerlantz, M., Bramnert, M., Manhem, P., Laurila, E., and Groo, L.C. 2001. Inhibition of the rise in FFA by acipimox partially prevents $\mathrm{GH}$ - induced insulin resistance in GH-deficient adults. J. Clin. Endocrinol. Metab. 86:5813-5818.

36. Kelly, D.E., Reilly, J.P., Veneman, T., and Mandarino, L.J. 1990. Effects of insulin on skeletal muscle glucose storage, oxidation, and glycolysis in humans. Am. J. Physiol. 258:E923-E928.

37. Hubinger, A., Knode, O., Susanto, F., Reinauer, H., and Gries, F.A. 1997. Effects of the carnitine-acyltransferase inhibitor etomoxir on insulin sensitivity, energy expenditure and substrate oxidation in NIDDM. Horm. Metab. Res. 29:436-439.

38. Stein, D.T., et al. 1996. Essentiality of circulating fatty acids for glucosestimulated insulin secretion in the fasted rat. J. Clin. Invest. 97:2728-2735.

39. Casanueva, F., Villanueva, L., Penalva, A., Vila, T., and Cabezas-Cerrato J. 1981. Free fatty acid inhibition of exercise-induced growth hormone secretion. Horm. Metab. Res. 13:348-350.

40. Samra, J.S., et al. 1998. Peripheral fat metabolism during infusion of an exogenous triacylglycerol emulsion. Int. J. Obes. Relat. Metab. Disord. 22:806-812.

41. Dobbins, R.L., et al. 1998. A fatty acid-dependent step is critically important for both glucose- and non-glucose-stimulated insulin secretion. J. Clin. Invest. 101:2370-2376.

42. Andreotti, A.C., et al. 1994. Acute pharmacologic blockade of lipolysis normalizes nocturnal growth hormone levels and pulsatility in obese subjects. Metabolism. 43:1207-1213. 